\title{
An Approach to Develop Fast and Efficient Routing for In-Network Aggregation
}

\author{
Vishal Vitthal Bandgar \\ Asst. Professor \\ SVERI's College of Engineering \\ (Polytechnic) \\ Pandharpur, Solapur
}

\author{
A. S. Bhatlavande \\ Asst. Professor \\ SVERI's College of Engineering \\ (Polytechnic) \\ Pandharpur, Solapur \\ Supriya A. Shegdar \\ Research Scholar \\ N. K. Orchid C O E, \\ Solapur
}

\author{
G.A. Fattepurkar \\ Asst. Professor \\ V.V.P. Polytechnic, \\ Solapur.
}

\begin{abstract}
Huge scale thick Remote Sensor Networks (Wsns) will be progressively sent in distinctive classes of uses for precise checking. Because of the high thickness of hubs in these networks, it is likely that excess information will be caught by adjacent hubs when sensing an occasion. Since vitality preservation is a key issue in Wsns, information combination and aggregation ought to be misused in request to spare vitality. For this situation, excess information can be accumulated at halfway hubs lessening the size and number of traded messages and, accordingly, diminishing correspondence expenses and vitality utilization. In this work, we propose a novel Information. Directing for In-System Aggregation, called DRINA, that has some key viewpoints, for example, a diminished number of messages for setting up a steering tree, expanded number of covering courses, high aggregation rate, and dependable information aggregation and transmission. The proposed DRINA calculation was widely contrasted with two other known arrangements: the Data Combination based Part Task (Infra) and Briefest Way Tree (SPT) calculations. Our results demonstrate obviously that the directing tree constructed by DRINA gives the best aggregation quality when contrasted with these different calculations. The acquired results demonstrate that our proposed arrangement beats these arrangements in diverse situations and in distinctive key perspectives needed by Wsns.
\end{abstract}

\section{Keywords}

Wireless sensor networks Routing protocol, in-network aggregation.

\section{INTRODUCTION}

Remote sensor networks are generally utilized as a part of numerous diverse applications, at first for military networks and in addition in different zones like environment, wellbeing, territory checking and business purposes. With the late achievement of "Micro Electro Mechanical Frameworks (MEMS)" technology [2] whereby sensors are getting to be littler and more adaptable, WSN guarantees a lot of people new application territories later on.

Information aggregation minimizes excess information which thusly diminishes the quantity of packets transmitted to the base station bringing about protection of vitality and data transmission. Since information aggregation transmits just the helpful or resultant data to the end point, the issue of system blockage, traffic implosion and cover can be overcome [3]. In exemplary flooding, hubs don't alter their exercises focused around the measure of vitality accessible to them at a given time. Information aggregation techniques make the hubs of the system to be "re-source-mindful", i.e. adjust their correspondence and processing to the condition of their vitality assets. The principle objective of information aggregation is to gather and "consolidate" information in a vitality proficient way so that the lifespan of the WSN is expanded. The configuration of a productive information aggregation calculation is a testing re-look issue.

In proposed methodology we concentrate on an alternate paramount part of sensor networks, specifically in-system aggregation and information administration. These techniques permit to exchange off correspondence for computational many-sided quality. Given the application territory, system asset demands, and the way that nearby reckoning regularly expends altogether less vitality than correspondence, Insystem information aggregation and administration are at the very heart of sensor system research. Specifically, asset effectiveness, convenient conveyance of information to the sink hub, and precision or granularity of the results is clashing objectives and the ideal tradeoff among them to a great extent relies on upon the particular application.

At first, In-system aggregation techniques included distinctive approaches to course packets to consolidate information originating from diverse sources however guided towards the same destination(s). As such, these conventions were essentially directing calculations which varied from more customary impromptu steering conventions in the metric they used to choose the directing ways. All the more as of late, numerous extra studies have been distributed, tending to the steering issue as well as systems to speak to and consolidate information all the more proficiently. In-system information aggregation is a complex issue that includes numerous layers of the convention stack and diverse parts of convention configuration, and a characterization and arrangement of ideas and calculations is even now ailing in the writing.

In this study, we have given a meaning of In-system information aggregation and distinguished its key components: information spread and inquiry instruments (with specific concentrate on the steering and Macintosh layer), aggregation capacities, and information structure. By its exceptionally nature, in-system aggregation concerns a few layers of the convention stack, and any effective arrangement is prone to oblige a cross-layer plan. On the other hand, we 
note that the vast majority of the current exploration concentrates on systems administration issues, for example, directing, frequently considering just exceptionally basic methodologies to total information. Also, much work still stays to be carried out to give cross-layer arrangements, representing application, information representation, steering and Macintosh viewpoints. Truth be told, the plans proposed so far regularly concentrate on just a subset of these viewpoints, ordinarily attempting to consolidation directing and information aggregation techniques, however disregarding Macintosh, application or information representation issues. At last, an alternate perspective still not profoundly examined concerns the memory and the computational assets permit to support information aggregation transforming.

Subsequently to overcome above said issues with existing techniques, as of late numerous different methodologies are exhibited as we considered in [1]. DRINA [1] as of late exhibited. However DRINA further needs to be explored and reached out by considering the key system execution parameters like normal throughput, end to end delay, bundle conveyance degree and the most essential element is normal vitality utilization. In proposed methodology we are further displaying the new form of this DRINA technique

Remote Sensor System (WSN) comprises of spatially disseminated self-sufficient gadgets that helpfully sense physical or natural conditions, for example, temperature, sound, vibration, weight, movement, or pollu- tants at distinctive areas [1], [2]. Wsns have been utilized as a part of uses, for example, ecological observing, country security, discriminating framework frameworks, correspondences, producing, and numerous different applications that can be basic to spare lives and resources [3], [4], [5].

Sensor hubs are vitality compelled gadgets and the vitality utilization is by and large connected with the measure of assembled data, since correspondence is frequently the most lavish action regarding vitality. Consequently, calculations and conventions outlined for Wsns ought to consider the vitality utilization in their origination [6], [7], [8], [9]. Also, Wsns are data-driven networks that typically deliver a lot of data that needs to be steered, regularly in a multihop style, to a sink hub, which acts as a door to an observing focus (Fig. 1). Given this situation, routing assumes a critical part in the data social event process.

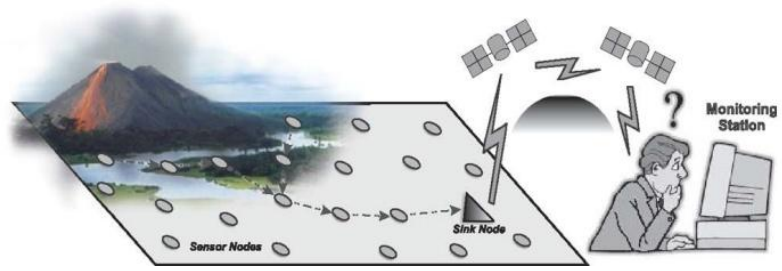

Fig. 1. Data aggregation aware routing, a key algorithm for data-driven WSNs

A conceivable technique to enhance the routing assignment is to utilize the accessible preparing limit gave by the between intercede sensor hubs along the routing ways. This is known as data-driven routing or in-system data aggrega- tion. For more proficient and successful data gathering with a base utilization of the constrained assets, sensor hubs ought to be arranged to adroitly report data by settling on neighborhood choices [10], [11], [12], [13]. For this, data aggregation is a powerful procedure for sparing vitality in Wsns. Because of the innate excess in crude data accumulated by the sensor hubs, in-systems administration aggregation can frequently be utilized to lessening the correspondence cost by wiping out redun- dancy and sending just littler collected informa- tion. Since insignificant correspondence heads straightforwardly to vitality investment funds, which augments the system lifetime, in-system data aggregation is a key technology to be underpinned by Wsns. In this work, the terms data combination and data aggregation are utilized as equivalent words. In this connection, the utilization of data combination is twofold [14]: 1) to exploit data excess and expand data exactness, and 2) to decrease correspondence load and spare vitality.

One of the principle challenges in routing calculations for Wsns is the manner by which to ensure the conveyance of the sensed data even in the vicinity of hubs disappointments and intrusions in correspondences. These disappointments get to be considerably more basic when data aggregation is performed along the routing ways since packets with totaled data contain informa- tion from different sources and, at whatever point one of these packets is lost a lot of data will likewise be lost. In the connection of WSN, data aggregation mindful routing conventions ought to present some attractive character- istics, for example, a diminished number of messages for setting up a routing tree, expanded number of covering courses, high aggregation rate, furthermore a dependable data transmission. Keeping in mind the end goal to defeat these difficulties, in this work, we propose a novel Data Routing calculation for In-System Aggregation for Wsns, which we allude to as DRINA calculation. Our proposed calculation was imagined to amplify data combination along the correspondence course in solid route, through a deficiency tolerant routing component.

\section{LITERATURE REVIEW}

In the first place change to a basic information aggregation capacity to consider the spatial relationship is displayed in [2]. In this procedure, the reliance on the separation among hubs is measured by a rot capacity which might, e.g., rot exponentially with an expanding jump separation [2]. Amid the information aggregation, each one perusing is weighed by a rotting element which diminishes with the separation to its source. The schema can be stretched out by furthermore representing transient and semantic connection. In any case, this remaining parts an open and for the most part unaddressed issue. Tina [3] Worldly coherency-mindful inSystem Aggregation [3] (Tina) deals with top of a steering tree (i.e., TAG or Cougar, see Area IV-A) having the information social event point (sink) as its root. It abuses the transient relationship in a succession of sensor readings to lessen vitality utilization by stifling those values that don't influence the normal nature of the collected information.

DADMA [4]data Aggregation and Weakening by Modulus Tending to (DADMA) [4] is a disseminated information aggregation and weakening system for sensor networks where hubs total or weaken sensed qualities as per the tenets given in a SQL articulation. DADMA treats a remote sensor arrange as a conveyed social database.

Information Aggregation by method for Criticism Control [5]. The creators of [5] characterize a methodology to tune the level of information aggregation while keeping up indicated dormancy limits on information conveyance and minimizing the vitality utilization. They consider time-obliged reference situations managing constant applications which force particular time obligations to the conveyance of sensor estimations. Information is gathered into diverse classes 
connected with distinctive limits on the conveyance time. The point is to ensure the conveyance of all information at the base vitality expense while fulfilling record-breaking stipulations. The information aggregation degree is adjusted likewise to meet these necessities.

Summary Dispersion System [6]: A late answer for the information aggregation issue has been proposed in [6]. The fundamental commitment of the paper is to characterize aggregation capacities and information structures which are vigorous to considering the same sensor readings in the information aggregation process numerous times (twofold including issue). This is essential when information aggregation is utilized as a part of conjunction with multi-way directing plans.

In [7], Creator has dissected the issue of timing when conglomerating information in Remote Sensor Networks. They have demonstrated, both through scientific investigation and reproduction that their proposed convention can spare vitality by diminishing the measure of information transmitted in a WSN.

In [8], this paper assesses the impact of timing in information aggregation calculations. In-system aggregation attains vitality proficient information spread by preparing information as it spills out of data sources to sinks. The fundamental objective was to demonstrate that the choice of when to "check out" information as it is transformed by hubs have noteworthy execution affect regarding information precision and freshness.

In [9], this paper creator model information driven directing and contrast its execution and customary end-to-end steering plans. It was analyzed the effect of source-objective arrangement and correspondence system thickness on the vitality expenses and deferral connected with information aggregation. It was demonstrated that information driven directing offers noteworthy execution picks up over an extensive variety of operational situations.

Since it is a NP-hard issue, a few heuristics for the Steiner tree issue can be found in the writing. Rough answers for the issue are displayed in [10] and [11]. Be that as it may, these arrangements are not fitting for asset obliged networks, for example, Wsns, since their appropriated execution oblige an extensive number of messages trade when setting up the directing tree and, subsequently, bringing about high vitality utilization

\section{PROBLEM ANALYSIS}

In WSNs generally high numbers of packets are transmitted to base station which may cause the problems of network congestion, traffic implosion. Thus to overcome these issues with existing techniques recently many other approaches are presented like Information Fusion-based Role Assignment (InFRA), Shortest Path Tree (SPT), TiNA[3], ADMA[4].But all the existing techniques have some merits and demerits. To overcome these problems we propose new approach DRINA.

The proposed approach will be extensively compare to two other known solutions: the InFRA and SPT algorithms. In the proposed approach we will check the performance of DRINA for data packets, overhead, tree cost as well as efficiency which can only define the efficiency of this method for network scalability, communication costs, aggregation rate, and delivery efficiency and aggregated data delivery rate as compared to existing one. However, along with just building efficient In-Network Aggregation method routing efficiency is also most important factor to decide the best method further.
There is still ambiguity about networking and routing performance of DRINA

\section{PROPOSED METHODOLOGY}

In-network aggregation is the global process of gathering and routing information through a multi-hop network, handling information at transitional hubs with the target of diminishing asset utilization (specifically vitality), in this manner expanding system lifetime. As stated in problem definition, DRINA is recent and most efficient technique for In-network aggregation for wireless sensor networks in terms of scalability, delivery efficiency, aggregation rate, and aggregated data delivery rate. DRINA will deliver the best performances against the existing methods like SPT. In this project spatial and temporal correlation of the aggregated data will also be taken into consideration as well as the construction of a routing tree that meets application needs. We also plans to modify the DRINA algorithm to stochastically select nodes that will be part of the communication structure.

We will further investigate the performances of DRINA approach by extending its analysis for routing efficiency. We will display the assessment of this proposed DRINA against the SPT regarding normal throughput, end to end delay, packet conveyance proportion and normal vitality utilization for versatile and differing system situations. Further in addition to this we will add the recent method for WSN security into the DRINA.

\subsection{Performance Evaluation}

Here we will assess the proposed DRINA calculation and contrast its execution. The Infra and SPT calculations. These two calculations were picked for being admirably known in the writing and have the same objectives that the proposed DRINA calculation. We assess the DRINA execution under the accompanying measurements:

1. $\quad$ packet conveyance rate.
2. $\quad$ control overhead.
3.
4. $\quad$ routing tree cost.
5. loss of crude data.
6. loss of accumulated data.
7. $\quad$ transmissions number.

\section{OBJECTIVES AND SCOPE}

The main aim of proposed approach is to present the investigation and analysis of DRINA method by considering all routing performance metrics against existing SPT method.

-To present the literature review over different approaches used for In-network aggregation for WSN

-To present the analysis of different methods based on their performance scalability, efficiency metrics

-To present and examine the proposed approach in points of interest with their building design

-To present the functional examination of proposed work and its assessment against the current strategies

\section{CONCLUSION AND FUTURE WORK}

Aggregation mindful routing calculations assume a vital part in occasion based Wsns. In this work, we exhibited the DRINA calculation, a novel and dependable Information 
Aggregation Mindful Routing Convention for Wsns. Our proposed DRINA calculation was broadly contrasted with two other known routing calculations, the Infra and SPT, in regards to scalability, communication costs, conveyance effectiveness, aggregation rate, and totaled information conveyance rate. By amplifying the aggregation focuses and offering a shortcoming tolerant instrument to enhance conveyance rate, the acquired comes about unmistakably demonstrate that DRINA beat the Infra and SPT calculations for all assessed situations. Additionally, we demonstrate that our proposed calculation has some key viewpoints needed by Wsns aggrega- tion mindful routing calculations, for example, a decreased number of messages for setting up a routing tree, augmented number of covering courses, high aggregation rate, and dependable information aggregation and transmission.

As future work, spatial and transient connection of the amassed information will likewise be looked into and additionally the development of a routing tree that addresses application needs. We additionally want to alter the DRINA calculation to stochastically select hubs that will be a piece of the commu- nication structure. The objective is to discover a harmony between the overhead and the nature of the routing tree. Likewise, new procedures will be contrived to control the sitting tight time for aggregator hubs focused around two criteria: normal separation of the occasion organizers, and spatial and semantics occasion relationship.

\section{REFERENCES}

[1] Leandro Aparecido Villas, Azzedine Boukerche, Heitor Soares Ramos, Horacio A.B. Fernandes de Oliveira, Regina Borges de Araujo, and Antonio Alfredo Ferreira Loureiro, "DRINA: A Lightweight and Reliable Routing Approach for In-Network Aggregation in Wireless Sensor Networks", IEEE TRANSACTIONS ON COMPUTERS, VOL. 62, NO. 4, APRIL 2013.

[2] E. Cohen and H. Kaplan, "Spatially-Decaying Aggregation Over a Network: Model and Algorithms," in ACM SIGMOD 2004, Paris, France, Jun. 2004.
[3] A. Sharaf, J. Beaver, A. Labrinidis, and K. Chrysanthis, "Balancing energy efficiency and quality of aggregate data in sensor networks," The VLDB Journal, vol. 13, no. 4, pp. 384-403, Dec. 2004.

[4] E. Cayirci, "Data Aggregation and Dilution by modulus addressing in wireless sensor networks," IEEE Communications Letters, vol. 7, no. 8, pp. 355-357, Aug. 2003.

[5] T. Abdelzaher, T. He, and J. Stankovic, "Feedback control of data aggregation in sensor networks," in IEEE CDC 2004, Atlantis, Paradise Island, Bahamas, Dec. 2004.

[6] S. Nath, P. B. Gibbons, Z. R. Anderson, and S. Seshan, "Synopsis Diffusion for Robust Aggregation in Sensor Networks," in ACM SenSys 2004, Baltimore, MD, US, Nov. 2004.

[7] F. Hu, X. Cao, and C. May, "Optimized Scheduling for Data Aggregation in Wireless Sensor Networks," Proc. Int'l Conf. Information Technology: Coding and Computing (ITCC '05), pp. 557-561, 2005.

[8] I. Solis and K. Obraczka, "The Impact of Timing in Data Aggregation for Sensor Networks," IEEE Int'l Conf. Comm.,vol. 6, pp. 3640-3645, June 2004.

[9] B. Krishnamachari, D. Estrin, and S.B. Wicker, "The Impact of Data Aggregation in Wireless Sensor Networks," Proc. 22nd Int'l Conf. Distributed Computing Systems (ICDCSW '02), pp. 575-578, 2002.

[10] J. Al-Karaki, R. Ul-Mustafa, and A. Kamal, "Data Aggregation in Wireless Sensor Networks-Exact and Approximate Algorithms," Proc. High Performance Switching and Routing Workshop (HPSR '04), pp. 241245, 2004.

[11] G. Robins and A. Zelikovsky, "Improved Steiner Tree Approximation in Graphs," Proc. 11th Ann. ACM-SIAM Symp. Discrete Algorithms (SODA '00), pp. 770-779, 2000. 